\title{
A New Modified Method for Inserting Iliosacral Screw versus the Conventional Method
}

\author{
Sina Javidmehr ${ }^{1,2}$, Mohammad Reza Golbakhsh², Babak Siavashi' ${ }^{2}$ Parham Talebian ${ }^{1}$, \\ Morteza Dehnokhalaji ${ }^{1}$, Mohammad Javad Zehtab ${ }^{2}$, Mohammadreza Bozorgmanesh ${ }^{1}$ \\ ${ }^{1}$ Sina Trauma and Surgery Research Center, Tehran University of Medical Sciences, Tehran, Iran \\ ${ }^{2}$ Department of Orthopedic Surgery, Sina Hospital, Tehran University of Medical Sciences, Tehran, Iran
}

\section{Study Design: Methodological study.}

Purpose: To our knowledge, this is the first study to introduce a new modified method for inserting iliosacral screws and to compare its results with those of a conventional method.

Overview of Literature: Previous techniques, such as open reduction and internal fixation, are associated with perioperative hemorrhage, postoperative infection, and neurological deficits. Although percutaneous iliosacral screw insertion confers the advantage of being minimally invasive, leading to less blood loss and a low postoperative infection rate, it harbors the risk of screw malpositioning due to narrow sacral proportions and a high interindividual variability.

Methods: Nine cadaveric pelvises were included in this study, with one hemipelvis of each being assigned to the new modified method and the other to the conventional iliosacral screw insertion method. In the new modified method, the guidewire entry point was determined using a lateral sacral X-ray. To do so, we first identified the anterosuperior quadrant of the S1 body on one hemipelvis. The anterosuperior quadrant was further divided into four imaginary quadrants, and the guidewire was inserted into the posteroinferior quadrant. The guidewire trajectory was perpendicular to the sagittal plane so that the guidewire resembled a single point in the lateral sacral view. Guidewires were inserted into corresponding hemipelves using the conventional method as described in the literature. Subsequently, an axial computed tomography scan with 1-mm fine cuts was obtained, and sagittal and coronal views were reconstructed. The distance of the guidewire from the sacral canal, anterior sacral cortex, and first sacral foramen was measured in axial, sagittal, and coronal views. The minimum measurement among different views was defined as the safety index of the insertion methods. The conventional and new modified methods were then compared in terms of safety and duration of the procedure.

Results: The minimum distance of the guidewire from the S1 foramen and anterior sacral cortex was not significantly different between the two methods. However, the minimum distance between the guidewire and sacral canal was significantly greater in the new modified method than in the conventional method. The duration of guidewire insertion was significantly shorter in the new modified method than in the conventional method.

Conclusions: This new modified method of iliosacral screw insertion could be safely and simply implemented while taking less surgical time than the conventional methods.

Keywords: Pelvic ring fractures; Sacroiliac screws; Sacral fractures

Received Mar 7, 2017; Revised Jun 5, 2017; Accepted Jun 6, 2017

Corresponding author: Mohammad Reza Golbakhsh

Department of Orthopedic Surgery, Sina Hospital, Tehran University of Medical Sciences, Imam Street, Tehran, Iran

Tel: +98-21-66705140, Fax: +98-21-66705511, E-mail: dmrgolbakhsh@yahoo.com 


\section{Introduction}

Iliosacral screw fixation is a minimally invasive method for treating unstable pelvic ring injuries. Because of the many complications, including pain, leg length difference, walking difficulties, and sexual impairment [1], associated with conservative treatments of these injuries, percutaneous iliosacral screw fixation is widely used and has become more popular among surgeons [2-4]. Sacroiliac joint dislocations, sacral fractures, iliac crescent fractures, and combinations of the above injuries are indications for this surgery [5].

Previous techniques, such as open reduction and internal fixation, are associated with perioperative hemorrhage, postoperative infection, and neurological deficits [1]. Although percutaneous iliosacral screw insertion confers the advantage of being minimally invasive, leading to less blood loss and a low postoperative infection rate $[1,6,7]$, it harbors the risk of screw malpositioning due to narrow sacral proportions and a high interindividual variability.

On the other hand, morbid obesity, intra-abdominal contrast, or excessive bowel gas may deteriorate the fluoroscopic image quality, especially while obtaining outlet and inlet pelvic views [8]. Avoiding these complications during surgery mandates a long surgical time and high exposure to radiation.

Many imaging techniques, including fluoroscopy or computed tomography (CT) guidance, intraoperative neurologic monitoring, and computer assisted fluoro-navigation [9], have been developed to be used during surgeries in order to minimize the incidence of screw malposition. Despite the precise imaging of the osseous structures provided by CT scan [8,10-14], the conventional fluoroscopyassisted method remains the most popular technique. In this technique, anteroposterior (AP) pelvic radiographs (inlet, outlet, and lateral sacral views) are used to determine the site and trajectory of screw insertion $[15,16]$. A radiolucent surgical table and intraoperative $\mathrm{C}$-arm fluoroscopy are required for obtaining these radiographs.

This cadaveric study aimed to introduce a simplified method that allows less experienced surgeons to insert an iliosacral screw, even when the quality of intraoperative fluoroscopic views is suboptimal. We developed the point of entry and trajectory of screw fixation and compared its accuracy to that of a conventional method using reconstructed CT images.

\section{Materials and Methods}

This experimental study was conducted at the Sina Hospital (affiliated hospital of Tehran University of Medical Sciences) between March 2104 and September 2016. Nine cadaveric pelvises were used, with one hemipelvis being assigned to the new modified method and the other to the conventional iliosacral screw insertion method (Fig. 1).

As there was no fracture or displacement, the situation simulated that after closed anatomic reduction. All insertions were performed by the same expert pelvic surgeon. Iliosacral guidewire insertions in the right hemipelves were performed using the conventional method, and those in the left hemipelves were performed using the new modified method. We used 2-mm guidewires to minimize potential metal artifacts during postoperative CT scans later on.

\section{Conventional method}

The bony landmarks corresponding to the lateral sacral radiographic projection are shown in the Fig. 2A. The posterior aspect of the $\mathrm{S} 1$ body corresponded to the anterior aspect of the spinal canal. The iliac cortical density corresponded to the sacral ala on which the L5 nerve rested. The great vessels are located anterior to the anterior cortex of the sacral promontory, and these could be perforated. The S1 foramen and nerve root are located in the residual disc space between S1 and S2. The entry point for the iliosacral screw, which should be below and behind the iliac cortical density, was confirmed on a lateral sacral view. A

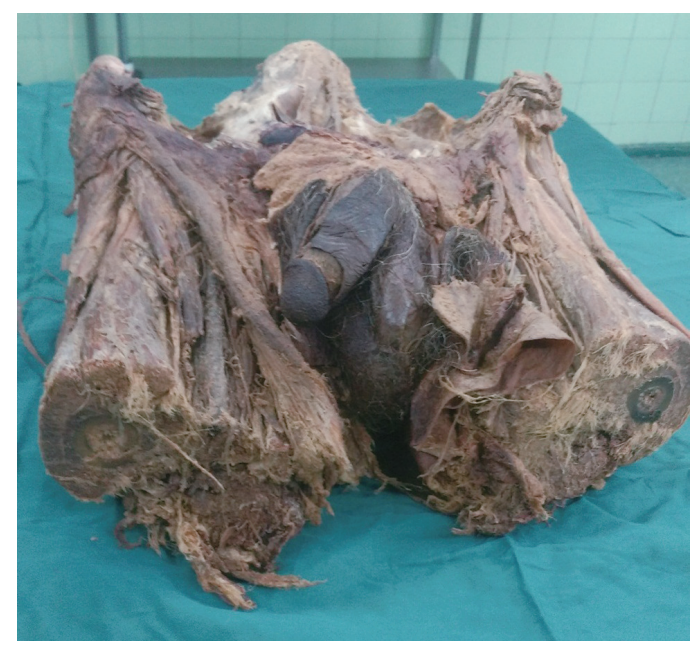

Fig. 1. One of the nine pelvic cadavers on the operating table. 


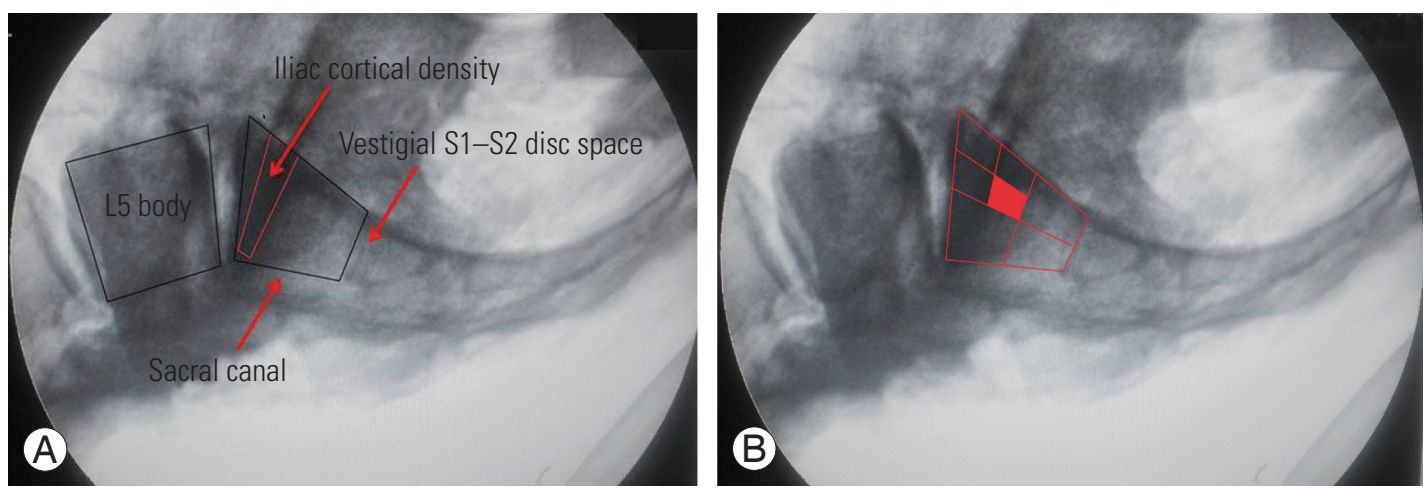

Fig. 2. (A) The bony landmarks corresponding to the lateral sacral radiographic projection (arrows). (B) Entry point in the new modified method.

2-mm guidewire was gently tapped into the iliac blade at the entry point so that it did not become dislodged during the subsequent procedure. The $\mathrm{C}$-arm was used to obtain inlet, outlet, and AP view images, and a guidewire was inserted to reach superior to the $\mathrm{S} 1$ foramen in the outlet view, below the L5-S1 intervertebral disc space in the AP view, and within the $S 1$ body in the inlet view [17]. If the guidewire trajectory compromised either the sacral foramen or spinal canal, the guidewire was removed and then correctly reinserted. In the aforementioned conventional method, the exact entry point and the guidewire trajectory were not defined, and the surgeon was allowed to use any entry point under iliac cortical density and any trajectory at his/her own discretion. The guidewire was advanced to the midline.

\section{The new modified method}

Based on our experience, we identified an entry point that was easier to find on lateral sacral views even on suboptimal intraoperative fluoroscopic X-rays. We first identified the anterosuperior quadrant of the S1 body and then inserted the screw in its posteroinferior quadrant (Fig. 2B). This point was supposed to be located below the iliac cortical density. In this method, we also attempted to insert the guidewire perpendicular to the sagittal plane so that the guidewire resembled a single point on a lateral sacral view (Fig. 3). In addition, in this new method we relied only on the lateral sacral view. The guidewire also was advanced to the midline on a pelvic AP view. We did not obtain outlet or inlet views in this method, and accurate insertion of the guidewire was later evaluated using postoperative reconstructed CT scan (Figs. 4, 5).

The duration of the procedures was measured for each

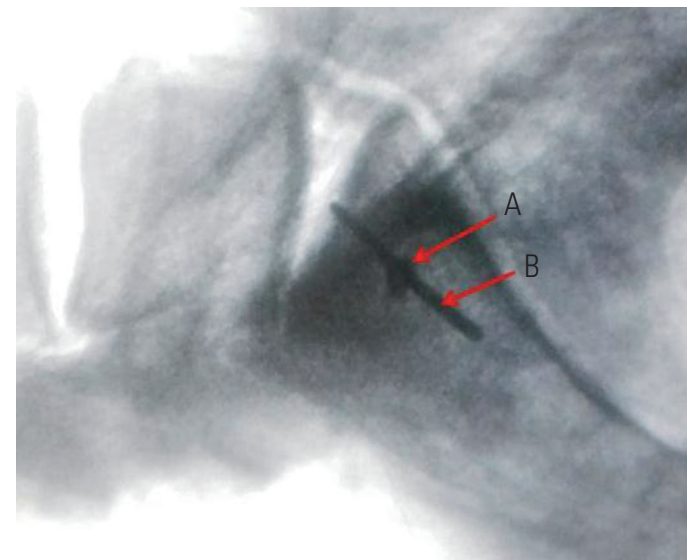

Fig. 3. Guidewire insertion in the conventional (a) and new modified (b) methods.

method. The nine cadaver pelvises were examined using reconstructed CT scan with fine (1-mm) slices. All reconstructed axial, sagittal, and coronal CT images were evaluated with image analysis software (ClearCanvas Workstation V7.1 SP1; ClearCanvas, Toronto, ON, Canada) to identify the minimum distance of guidewires from the sacral canal, anterior sacral cortex, and S1 foramen. For example, the minimum distance measurements for cadaver pelvis number 2 are shown in Figs. 6-8. The potential penetration of the guidewire into each of these anatomical areas was also evaluated.

All data were analyzed using IBM SPSS software ver. 22.0 (IBM Corp., Armonk, NY, USA). Data are presented as mean \pm standard deviation and median (interquartile range [IQR]) for continuously distributed variables when appropriate. The Mann-Whitney $U$-test was used to test the statistical significance of the differences in the safety index and the duration of procedures. A $p$-value of $<0.05$ was considered significant. 

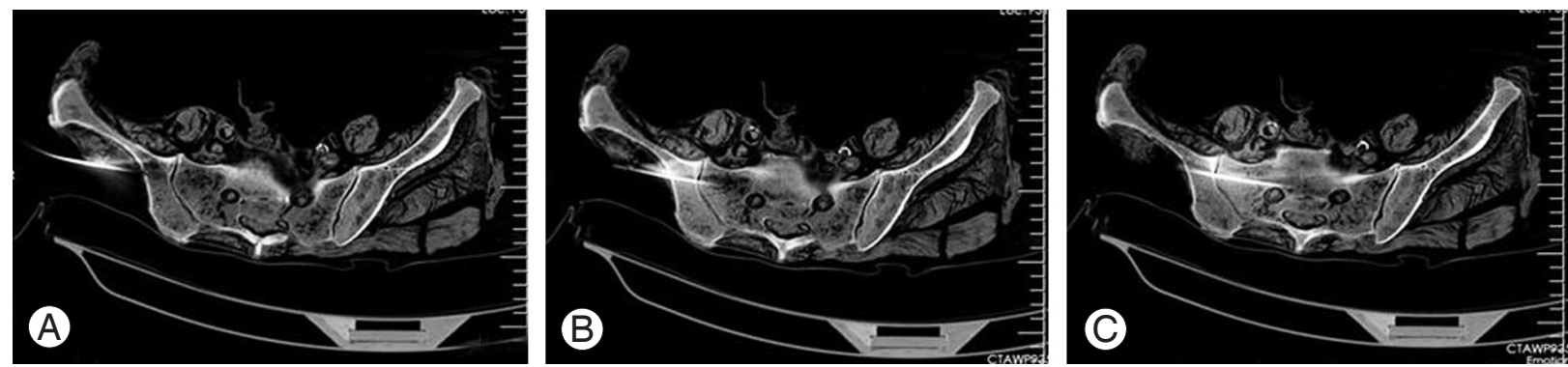

Fig. 4. (A-C) Guidewire trajectory in the conventional method was evaluated by postoperative computed tomography scan.
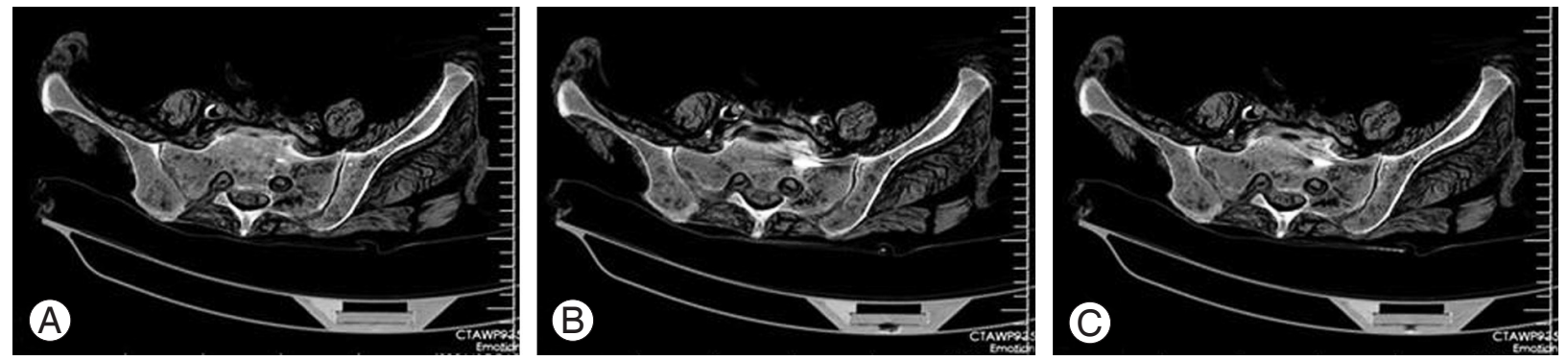

Fig. 5. (A-C) Guidewire trajectory in the new modified method was evaluated by postoperative computed tomography scan.
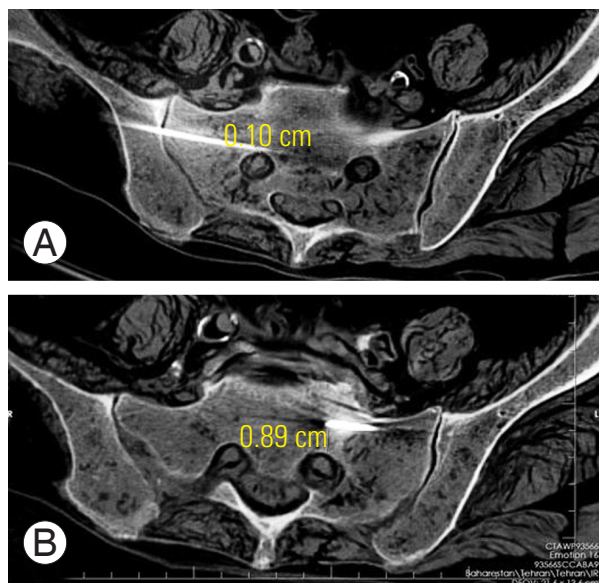

Fig. 6. (A) Pelvis number 2: right side, conventional method; minimum distance from the $\mathrm{S} 1$ foramen: $1 \mathrm{~mm}$. (B) Pelvis number 2: left side, new modified method; minimum distance from the $\mathrm{S} 1$ foramen: 8.9 $\mathrm{mm}$

\section{Results}

We evaluated 18 hemipelves (nine right and nine left hemipelves). The characteristics of the new modified and conventional methods are compared in Tables 1 and 2.

Median surgical duration was significantly shorter for the new modified method (median, 16; IQR, 12.5-18.5) than for the conventional method (median, 22; IQR, $18.5-26.5 ; p=0.005]$. The median magnitude of minimum distance from the sacral canal was greater in the new
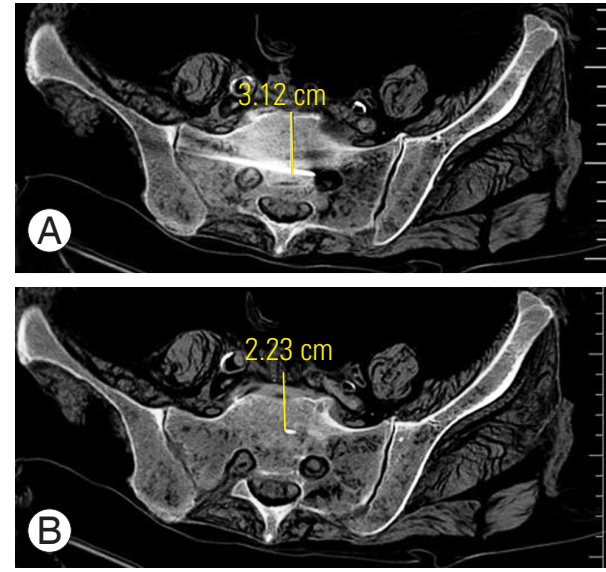

Fig. 7. (A) Pelvis number 2: right side, conventional method; minimum distance from the anterior sacral cortex: $31.2 \mathrm{~mm}$. (B) Pelvis number 2: left side, new modified method; minimum distance from the anterior sacral cortex: $22.3 \mathrm{~mm}$.
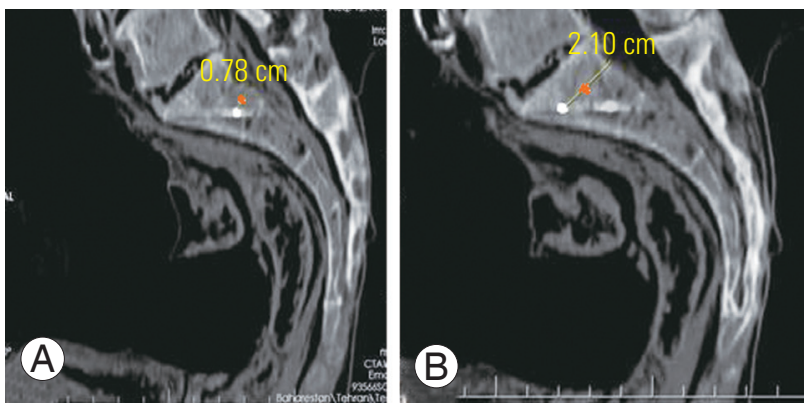

Fig. 8. (A) Pelvis number 2: right side, conventional method; minimum distance from the sacral canal: $7.8 \mathrm{~mm}$. (B) Pelvis number 2: Ift side, new modified method; minimum distance from the sacral canal: $21 \mathrm{~mm}$. 
Table 1. Characteristics of the new-modified vs. conventional methods

\begin{tabular}{|c|c|c|c|c|c|c|c|c|}
\hline \multirow[b]{2}{*}{ Pelvic no. } & \multicolumn{2}{|c|}{$\begin{array}{l}\text { Distance from sacral } \\
\text { canal (mm) }\end{array}$} & \multicolumn{2}{|c|}{$\begin{array}{l}\text { Distance from S1 } \\
\text { foramen (mm) }\end{array}$} & \multicolumn{2}{|c|}{$\begin{array}{l}\text { Distance from ant. } \\
\text { sacral cortex (mm) }\end{array}$} & \multicolumn{2}{|c|}{$\begin{array}{l}\text { Time } \\
\text { (min) }\end{array}$} \\
\hline & $\begin{array}{l}\text { Conventional } \\
\text { method }\end{array}$ & $\begin{array}{c}\text { New } \\
\text { modified } \\
\text { method }\end{array}$ & $\begin{array}{l}\text { Conventional } \\
\text { method }\end{array}$ & $\begin{array}{l}\text { New } \\
\text { modified } \\
\text { method }\end{array}$ & $\begin{array}{l}\text { Conventional } \\
\text { method }\end{array}$ & $\begin{array}{c}\text { New } \\
\text { modified } \\
\text { method }\end{array}$ & $\begin{array}{l}\text { Conventional } \\
\text { method }\end{array}$ & $\begin{array}{c}\text { New } \\
\text { modified } \\
\text { method }\end{array}$ \\
\hline 1 & 1.1 & 10.7 & 2 & 2 & 13.3 & 18.1 & 20 & 12 \\
\hline 2 & 7.8 & 21 & 1 & 8.9 & 31.2 & 22.3 & 18 & 10 \\
\hline 3 & 6.4 & 15 & 3 & 5.9 & 17 & 19.3 & 25 & 14 \\
\hline 4 & 2.6 & 12 & 6 & 6.7 & 19 & 22 & 22 & 13 \\
\hline 5 & 4 & 9 & 2 & 2 & 24 & 7.1 & 14 & 16 \\
\hline 6 & 3.9 & 7.6 & 6.7 & 1 & 7 & 12 & 19 & 19 \\
\hline 7 & 5 & 18 & 1 & 1.4 & 9 & 16.5 & 30 & 21 \\
\hline 8 & 8.4 & 12 & 7.3 & 2.2 & 13.4 & 19.5 & 26 & 18 \\
\hline 9 & 5 & 5.4 & 2.8 & 5 & 11.1 & 14 & 27 & 17 \\
\hline
\end{tabular}

Table 2. Summary of the characteristics of the new modified vs. conventional method

\begin{tabular}{|c|c|c|c|}
\hline Variable & Mean \pm standard deviation & Median (interquartile range) & $p$-value for medians \\
\hline Distance to $\mathrm{S} 1$ foramen (mm) & & & 0.4 \\
\hline Conventional method & $3.5 \pm 2.4$ & $2.8(1.5-6.4)$ & \\
\hline New modified method & $3.9 \pm 2.8$ & $2.2(1.5-6.4)$ & \\
\hline Distance to sacral canal (mm) & & & 0.03 \\
\hline Conventional method & $5 \pm 2.3$ & $5(3.3-7.0)$ & \\
\hline New modified method & $12.3 \pm 4.9$ & $12(8.3-16.5)$ & \\
\hline Distance to anterior sacral cortex (mm) & & & 0.1 \\
\hline Conventional method & $16.1 \pm 7.6$ & $13.4(10.0-21.5)$ & \\
\hline New modified method & $16.7 \pm 4.9$ & $18.1(13.0-20.8)$ & \\
\hline Time (min) & & & 0.005 \\
\hline Conventional method & $18.9 \pm 5.5$ & $22(18.5-26.5)$ & \\
\hline New modified method & $15.5 \pm 3.5$ & $16(12.5-18.5)$ & \\
\hline
\end{tabular}

modified method than in the conventional method (12.0 [IQR, 8.3-16.5] versus 5.0 [IQR, 3.3-7.0]; $p=0.03$ ). The median magnitude of minimum guidewire distance from first sacral foramen was not statistically different between the new modified and conventional methods (2.2 [IQR, $1.5-6.4]$ versus 2.8 [IQR, $1.5-6.4] ; p=0.4]$. The median magnitude of the minimum distance from the anterior sacral cortex was not statistically different between the new modified and conventional methods (18.1 [IQR, 13.0-20.8] versus 13.4 [IQR, 10.0-21.5]; $p=0.1$ ). Regardless of the method used, the sacral canal, the anterior cortex, and the S1 foramen were not penetrated during guidewire insertion.

\section{Discussion}

We developed a new iliosacral screw insertion method that was found to be safer and faster to implement than its conventional counterpart. The new modified and conventional methods were similar in terms of the safety index for distance from the anterior cortex and S1 foramen. However, the new modified method was found to be safer in terms of the distance from the sacral canal. Furthermore, the new modified method was easier and faster to 
implement than the conventional method. In both methods, the sacral canal, anterior cortex, and S1 foramen were not penetrated during guidewire insertion.

In a previous study conducted by Giannoudis et al. [16], plastic pelvis were assigned to two groups: group A, insertion of the screws on the right side of the model using inlet and outlet views, and group B, insertion of the screws on the left side of the model using inlet, outlet, and lateral views. After insertion into S1, there were three cases of foraminal and none of central canal perforation in both groups. However, after S2 insertion, the investigators observed 10 foraminal and five canal perforations in both groups. A higher incidence of S1 screw misplacement was found in group A than in group B $(p=0.001)$. The investigators concluded that percutaneous screw fixation using inlet, outlet, and lateral views are preferable to using only inlet and outlet views [16].

Percutaneous iliosacral screw insertion is a minimally invasive technique that provides mechanical stability [3]. It can be used in patients with multiple trauma and in those who are hemodynamically unstable [18]. This technique confers stable fixation with negligible blood loss or soft-tissue damage $[19,20]$. Furthermore, it is less likely to cause neurovascular injury [8].

Precise understanding of anatomical landmarks and the quality of fluoroscopic imaging play an important role in the accurate placement of screws [16]. Identification of the optimal insertion point is crucial.

Some investigators have recommended iliosacral screw insertion under CT guidance. The application of CT scanning requires patients to be hemodynamically stable. Manipulation for reduction can be cumbersome if it is to be performed with CT scanning [17].

Our results should be interpreted in the context of the limitations. First, the sample size was small. Second, it was an in vitro study, so the results might not be directly extrapolated to the human population, although there is no evidence to support the notion that screw insertion into a distorted cadaveric pelvis can be more precise than that in humans. Third, we used guidewires instead of screws. Thus, the safety margins might have been narrower for definitive fixation than those reported herein. Fourth, we could not obtain CT scans with metal artifact reduction. Although this new modified method is safe and simple and takes less surgical time than the conventional method, it cannot definitively exclude the necessity of obtaining intraoperative outlet and inlet views as the consequences of iliosacral screw malpositioning can be devastating.

\section{Conclusions}

We developed a new iliosacral screw insertion method. The new modified and conventional methods were similar in terms of the safety index for distance from the anterior cortex and S1 foramen. However, the new modified method was safer in terms of the distance from the sacral canal. Furthermore, the new modified method was easier and faster to implement than the conventional method. Future studies must examine whether the findings of this cadaveric study can be reproduced in real-world situations.

\section{Conflict of Interest}

No potential conflict of interest relevant to this article was reported.

\section{Acknowledgments}

The authors would like to acknowledge the encouragement provided by Dr. Qolamreza Hasanzadeh. We would also like to thank the Department of Anatomy for the time and effort they put into preparing the cadaveric pelvis.

\section{References}

1. Van den Bosch EW, van Zwienen CM, van Vugt AB. Fluoroscopic positioning of sacroiliac screws in 88 patients. J Trauma 2002;53:44-8.

2. Matta JM, Saucedo T. Internal fixation of pelvic ring fractures. Clin Orthop Relat Res 1989;(242):83-97.

3. Routt ML Jr, Kregor PJ, Simonian PT, Mayo KA. Early results of percutaneous iliosacral screws placed with the patient in the supine position. J Orthop Trauma 1995;9:207-14.

4. Routt ML Jr, Simonian PT. Closed reduction and percutaneous skeletal fixation of sacral fractures. Clin Orthop Relat Res 1996;(329):121-8.

5. Griffin DR, Starr AJ, Reinert CM, Jones AL, Whitlock S. Vertically unstable pelvic fractures fixed with percutaneous iliosacral screws: does posterior injury pattern predict fixation failure? J Orthop Trauma 2003;17:399-405.

6. Templeman D, Goulet J, Duwelius PJ, Olson S, Da- 
vidson M. Internal fixation of displaced fractures of the sacrum. Clin Orthop Relat Res 1996;(329):180-5.

7. Shuler TE, Boone DC, Gruen GS, Peitzman AB. Percutaneous iliosacral screw fixation: early treatment for unstable posterior pelvic ring disruptions. J Trauma 1995;38:453-8.

8. Routt ML Jr, Simonian PT, Mills WJ. Iliosacral screw fixation: early complications of the percutaneous technique. J Orthop Trauma 1997;11:584-9.

9. Zhang J, Hamilton R, Li M, et al. Evaluation of partial cut-out of sacroiliac screws from the sacral ala slope via pelvic inlet and outlet view. Spine (Phila Pa 1976) 2015;40:E1264-8.

10. Duwelius PJ, Van Allen M, Bray TJ, Nelson D. Computed tomography-guided fixation of unstable posterior pelvic ring disruptions. J Orthop Trauma 1992;6:420-6.

11. Ebraheim NA, Coombs R, Jackson WT, Rusin JJ. Percutaneous computed tomography-guided stabilization of posterior pelvic fractures. Clin Orthop Relat Res 1994;(307):222-8.

12. Ebraheim NA, Coombs R, Rusin JJ, Hoeflinger MJ, Jackson WT. Percutaneous CT-guided stabilization of complex sacroiliac joint disruption with threaded compression bars. Orthopedics 1992;15:1427-30.

13. Ebraheim NA, Rusin JJ, Coombs RJ, Jackson WT, Holiday B. Percutaneous computed-tomographystabilization of pelvic fractures: preliminary report. J Orthop Trauma 1987;1:197-204.
14. Jacob AL, Messmer P, Stock KW, et al. Posterior pelvic ring fractures: closed reduction and percutaneous CT-guided sacroiliac screw fixation. Cardiovasc Intervent Radiol 1997;20:285-94.

15. Routt ML Jr, Nork SE, Mills WJ. Percutaneous fixation of pelvic ring disruptions. Clin Orthop Relat Res 2000;(375):15-29.

16. Giannoudis PV, Papadokostakis G, Alpantaki K, Kontakis G, Chalidis B. Is the lateral sacral fluoroscopic view essential for accurate percutaneous sacroiliac screw insertion?: an experimental study. Injury 2008;39:875-80.

17. Shrestha D, Dhoju D, Shrestha R, Sharma V. Percutaneous ilio-sacral screw fixation in supine position under fluoroscopy guidance. Kathmandu Univ Med J (KUMJ) 2015;13:56-60.

18. Abdelgawad AA, Davey S, Salmon J, Gurusamy P, Kanlic E. Ilio-Sacral (IS) screw fixation for sacral and Sacroiliac Joint (SIJ) injuries in children. J Pediatr Orthop 2016;36:117-21.

19. Collinge C, Coons D, Aschenbrenner J. Risks to the superior gluteal neurovascular bundle during percutaneous iliosacral screw insertion: an anatomical cadaver study. J Orthop Trauma 2005;19:96-101.

20. Hinsche AF, Giannoudis PV, Smith RM. Fluoroscopy-based multiplanar image guidance for insertion of sacroiliac screws. Clin Orthop Relat Res 2002;(395):135-44. 